\title{
Lower lid tarsal ectropion correction by three tarsoconjunctival spindles
}

\begin{abstract}
Lower eyelid tarsal ectropion is a clinical entity characterized for complete eversion of the tarsal plate. Its etiology is due the desinsertion of the lower eyelid on the tarsal plate. We present a case of chronic left lower eyelid tarsal ectropion in a 50 -year-old man. We performed three tarsoconjunctival spindles at $4 \mathrm{~mm}$ from the free edge, with a vertical diameter of $3 \mathrm{~mm}$ and horizontal of $4 \mathrm{~mm}$ distributed along the lower eyelid, with the nasal under the punctum. A double armed 6-0 vicryl suture was used which included conjunctivaretractors by the inferior border of the diamond, and by tarso-conjunctiva by the upper border. Once passed the suture is introduced through the center of the diamond to exit at the bottom groove of the lower eyelid. When the two ends are tied, the punctum and medial margin invert..In this case, the use of three tarsoconjunctival spindles alone was a favorable option, without complications, and with acceptable functional and cosmetic results. The aim of the treatment is to restore anatomy, functional and cosmetic of the patients. At 5 year follow-up, there have been no recurrences and the patient was symptom-free, without treatment.
\end{abstract}

Keywords: tarsal ectropion, tarsoconjunctival spindles, tarsal strip, lower eyelid
Volume 7 Issue 4 - 2017

\author{
Rafael Corredor-Osorio, Buitrago-Corredor \\ Vanessa Gabriela, Massaro-Corredor Martha \\ Gabriela \\ Service Oculoplastic and Orbit, Centro Ocular Corredor \\ Oftalmologia Especializada,Venezuela \\ Correspondence: Rafael Corredor-Osorio, Centro Ocular \\ Corredor Oftalmologia Especializada, Av. Bolívar, CC las Acacias \\ local 3I,Valera (Trujillo),Venezuela, Tel 58-27I-23I057I, \\ Email raficorredor@yahoo.com
}

Received: September 24, 2017 | Published: October 04, 2017

\section{Introduction}

Ectropion is a turning outward of the free border of the eyelid, resulting in exposure of the conjunctiva. ${ }^{1}$ It is classified in the following categories: congenital and acquired, which may be involutional, paralytic, cicatricial and mechanical. ${ }^{2,3}$ Tarsal ectropion is a clinical condition characterized by lower lid eversion which is limited to the tarsal-containing portion alone. ${ }^{4,5}$ It differs from ordinary senile ectropion in that does not progress beyond the tarsus and there is no relaxation of the tissues of the lid below the tarsus. It is similar to senile ectropion in that it is seen only in middle and old age and I be unilateral or bilateral. ${ }^{4} \mathrm{~A}$ big variety of factors isolated or combined leading the appearance of the lower lid ectropion include horizontal laxitud of the eyelid, vertical a shortening of the anterior lamella of the eyelid, paralysis of the orbicularis oculi muscle, desinsertion of the lower eyelid retractors and tumor near of the eyelid margin mechanically evert the eyelid. ${ }^{2,3}$

\section{Case report}

A 53 year-old man presented to our clinic with a constant left lower lid tarsal ectropion with severely injected, hypertrophied conjunctiva and epiphora. He gave history of schizophrenia and periocular dermatitis. Corrected visual acuity was 20/20 in both eyes. External examination revealed left upper eyelid ptosis, periocular dermatitis and blepharitis. There was no significant horizontal laxitud of lower lid, as well as of the lateral canthal tendon and no evidence of anterior lamella cicatrisation. The everted tarsus was marked edema and inflammation of the lower tarsal conjunctiva (Figure 1). There was no evidence of lid ulcers, horizontal lid laxity and enophthalmos. Bells phenomenon and ocular motility was normal. The patient underwent a left lower lid correction by three tarsoconjunctival spindles without lateral tarsal strip.

\section{Operative procedure}

Operation under local anesthesia with intravenous sedation and surgical microscope. Local anesthetic is infiltrated in the anterior and posterior lamella of the left lower eyelid. We performed by Transconjunctival approach, three tarsoconjunctival spindles, at $4 \mathrm{~mm}$ from the free edge, distributed along lower eyelid. Westcott scissors are then used to excise an diamond shaped piece of conjunctiva and retractors, with a vertical diameter of $3 \mathrm{~mm}$ and horizontal of $4 \mathrm{~mm}$ distributed along the lower eyelid, making the nasal under the punctum. Next, a double armed 5-0 polyglactin suture is passed through the inferior edge, which included retractors-conjunctiva, and tarso- conjunctiva by superior edge. Then, the needles are passed full thickness out through the center of the diamond to exit at the bottom groove of the lower eyelid in a slightly downward vector. When, the two ends are tied, on the skin over bolsters, punctum and medial marginal invert (Figure $2 \& 3$ ). Topical antibiotic ointment is applied twice daily for 7 days. The bolsters and sutures are removed in 10 days (Figure 4). At follow up 5 years postoperatively the cosmetic appearance of the eyelid is very satisfactory and there was no recurrence of the tarsal ectropion.

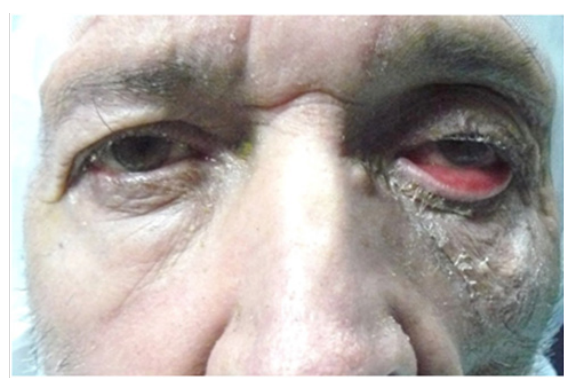

Figure I Preoperative photo showing severe tarsal left lower eyelid ectropion, facial dermatitis and blepharitis. 


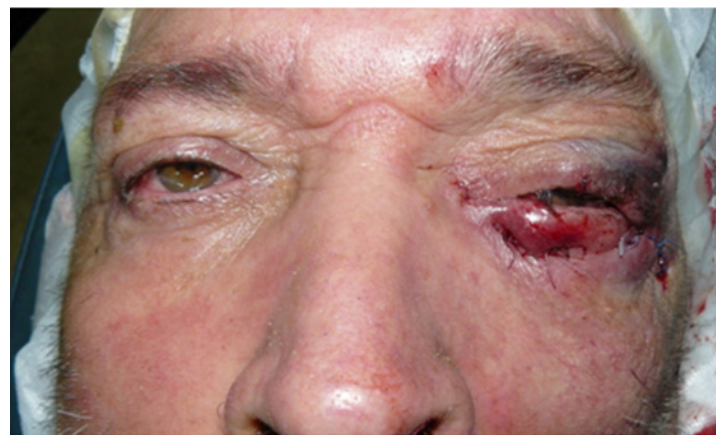

Figure $\mathbf{2}$ Three sutures in place with lid properly inverted immediately after surgery.

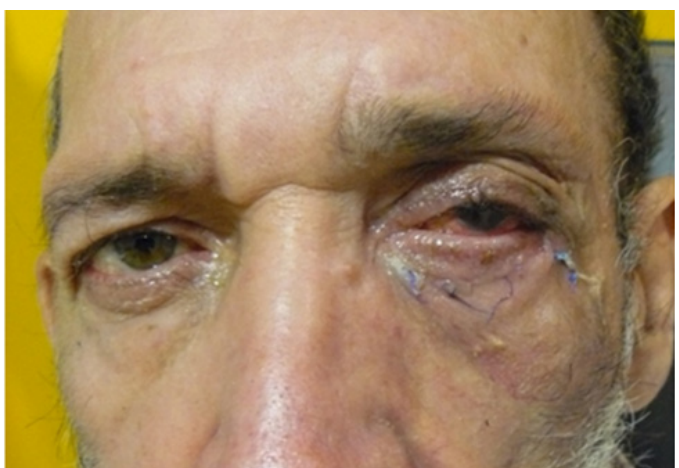

Figure 3 Postoperative photo shows significant improvement in left lowe position.

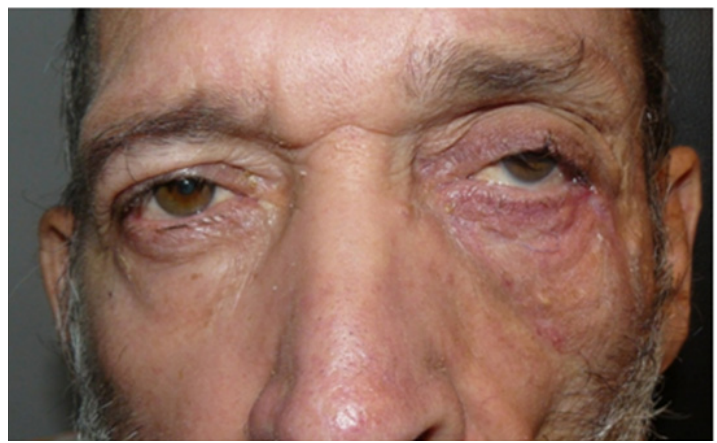

Figure 4 One month after transconjunctival approach retractors reinsertion.

\section{Discussion}

Ectropion of the lower eyelids occurs most often in the elderly, since senile or involutional ectropion is seen more frequently than all other types of ectropion combined. ${ }^{1}$ Severe lower eyelid ectropion is a surgical challenge. Lower eyelid tarsal ectropion is an unusual form of eyelid malposition, in which the entire lid is everted. The cause is desinsertion of the lower eyelid retractors, the capsulopalpebral fascia, from the inferior tarsal border. ${ }^{2,6,7}$ However, in patients with tarsal ectropion the tractional forces from the anterior lamella will evert the entire tarsus.

The desinsertion of the lower eyelid retractors from the inferior tarsal border may result in deepening of the lower fornix as the disinserted retractors pull the fornix down. Moreover, the fornix can also be shallower than expected and we believe this occurs if the fibers between the conjunctiva and retractors have also dehisced. The edge of the retractors may be seen through the conjunctiva as a grey band, but this may be difficult to assess clinically with chronic conjunctival changes, such as hyperemia and keratinization. ${ }^{2,8}$ Other clinical include medial tarsal ectropion (in absence of horizontal eyelid laxitud), loss of eyelid excursion in down gaze and loss of the lower eyelid skin crease. ${ }^{8}$ In the early stages is slight eversion of the punctum with epiphora giving the appearance of an early ordinary senile ectropion. However, even a cursory examination will show that there is no laxity of the lid tissues; in fact the skin of the lid is tense and has no "give". In the advanced stages the upper portion is everted on the lower portion of the lid like a cuff on a sleeve. Manual reinversion is always unsuccessful. Patients with ectropion have larger-than-average tarsal plates for their ages. ${ }^{6}$ The most common surgical technique for significant involutional ectropion repair is horizontal tightening with a lateral tarsal strip procedure. ${ }^{9}$ Until now, a variety of surgical techniques have been described for correction of medial ectropion: the lazy-T procedure, a triangle of posterior lamella including retractors is excised, with medial horizontal eyelid shortening. ${ }^{8}$ The medial spindle, procedure involves the excision of a diamond of conjunctiva and retractors with sutures tied anteriorly on the skin., ${ }^{2,10}$ A transconjunctival approach retractor plication and lateral tarsal strip. ${ }^{8}$ The repair of disinserted retractors to correct tarsal ectropion has been used anteriorly. Such alternative procedures are: an anterior approach via the skin to the retractors, following horizontal eyelid shortening. ${ }^{8}$ A posterior transconjunctival approach with excision of redundant conjunctiva following reattached of retractors to the inferonasal border with horizontal eyelid tightening procedure. ${ }^{8,11}$ A transconjunctival approach included the retractors with the inferior tarsal border, either alone, or in conjunction with horizontal eyelid tightening procedure. ${ }^{?}$

The case presented in this report lacked significant horizontal eyelid laxity changes. In this context, we hypothesize that reinserting the retractors with the inferior tarsal border and the use of inverting sutures would provide adequate support for the tarsus and resolve the tarsal ectropion. Moreover, the formation of an inflammatory cicatrix induced by the sutures also contributed to maintain the lid in an upright posture allowing the lower eyelid to heal properly and in good position, without fixation lateral canthal tendon. ${ }^{7}$ Thru, a nasal conjunctival spindle was practicing in order of reinsertion the retractors ${ }^{2,10}$ and we also used the same surgical technique at the medial and temporal region of the lower eyelid. ${ }^{2}$

\section{Conclusion}

The procedure described is relatively easy, rapid and safer method for disinserted retractors. According to our case report, the three tarsoconjunctival spindles procedure proved to be effective during an average follow up of nearly 5 years and therefore can be considered an appropriate technique for patients suffering tarsal ectropion.

\section{Informed consent}

Written informed consent was obtained from the patient for publication of this case report and accompanying images.

\section{Conflicts of interest}

Alll authors declare their no conflicts of interest for the publication of this article.

\section{Funding}

None. 


\section{References}

1. Siegel RJ. Severe ectropion: repair with a modified Tripier flap. Plast Reconstr Surg. 1987;80(1):21-28.

2. Diaz-Arriaga J, Perez-Alvarez J, Medina-Rivero F, et al. Tarsal ectropion correction by tarsal strip and three tarsoconjunctival spindles. Arch Soc Canar Oftal. 2008;19:81-84.

3. Miletic D, Elabjer KB, Bosnar D, et al. Our approach to operative treatment of lower lid ectropion. Acta Clin Croat. 2010;49(3):283-287.

4. Fox SA. Marginal (tarsal) ectropion. Arch Ophthalmol. 1960;63(4):660662.

5. Garza RM, Lee GK, Press BH. Tarsal ectropion repair and lower blepharoplasty: a case report and review of literature. $J$ Plast Reconstr Aesthet Surg. 2012;65(2):249-251.

6. Gupta B, Parmar B, Raina J, et al. Acute idiopathiclower lid ectropion. Indian J Ophthalmol. 2006;54(3):212-214.
7. Tse DT, Kronish JW, Buus D. Surgical correction of lower eyelid tarsal ectropion by reinsertion of the retractors. Arch Ophthalmol. 1991;109(3):427-431.

8. Fong KC, Mavrikakis I, Sagili S, et al. Correction of involutional lower eyelid medial ectropion with transconjunctival approach retractor placation and lateral tarsal strip. Acta Ophthalmol Scan. 2006;84(2):246249.

9. Kahana A, Lucarelli MJ. Adjunctive transcanthomy lateral suborbicularis fat lift and orbitomalar ligament resuspension in lower eyelid ectropion repair. Ophthal Plast Reconstr Surg. 2009;25(1): 1-6.

10. Nowinski TS, Anderson RL. The medial spindle procedurefor involutional medial ectropion. Arch Ophthalmol. 1985;103(111):1750-1753.

11. Wesley RE. Tarsal ectropion from detachment of the lower eyelid retractors. Am J Ophthalmol. 1982;93(4):491-495. 